\title{
A NOVEL DESIGN SPECIFICATION OF INTELLIGENT FISH FEEDING SYSTEM BASED ON SINGLE CHIP MICROCOMPUTER
}

\author{
Xuan Zhou, Zhangqing Yu, Zhongyuan Ge, Fanbin Meng, \\ Fanzhi Kong and Jianqiang Gao
}

School of Medical Information Engineering, Jining Medical University, Shandong 276826, P. R. China

\begin{abstract}
With the development of electronic technology, the micro-control technology has been developed rapidly. Nowadays, most people are pursuing high quality life, such as many people will use fish tanks to raise fish at home. But, most people spend very little time at home. Hence, there are some questions, for example, how to feed the ornamental fish at home, how to change water and clean up the aquarium and so on. In order to solve these problems, in this paper, we have designed a micro-controller-based intelligent fish tank control system.
\end{abstract}

Keywords: single chip microcomputer, intelligent fish feeding system.

\footnotetext{
${ }^{*}$ Corresponding author.

E-mail address: jianqianggaohh@126.com (Jianqiang Gao).
}

Copyright (C) 2020 Scientific Advances Publishers 2020 Mathematics Subject Classification: 68W25.

Received May 18, 2020 


\section{Introduction}

Many years ago, the fish tank was just a container for raising fish and seagrass. The most primitive fish tank was a simple structure of the water tank, and the aquarium did not have any complicated functions. Almost all families have been powered by electricity since the First World War, and aquariums are more widely welcomed [1]. The improvement of electric power has enabled the development of aquarium technology, making artificial lighting [2], ventilation [3], filtration [4], and water temperature heating [5]. The emergence of air transport has made it possible for more distant foreign species to enter, and has also made aquarium breeding more popular [6]. Nowadays, there are a large number of aquarium enthusiasts around the world.

In China, the development of aquarium control system is late [7]. With the rapid development of economic level, the rise of decoration industry, the people pursuit the good life more and more attention. So, the leisure, home decoration and other industries are booming. In recent years, the aquarium industry is born under the needs of human beings, and its rapid development has become a new economic force by the economic community and the industry concern. According to the latest information of aquarium products, the annual growth rate of the aquaculture industry scale reached $13.8 \%$ [8]. With the rapid development of aquarium products, the demand for the huge aquarium market has also greatly contributed to the development and production of various aquarium control equipment.

Research has suggested that ornamental fish have higher requirements for the surrounding environment of life, such as, the water temperature, oxygen content, water turbidity, and drug concentration are required to maintain a certain range [9]. Hence, the price of intelligent control fish tank is higher and higher. Generally speaking, the design of the smart fish tank has not been popularized and the cost of intelligent fish tank is high. So, the aquarium to maintain the living environment of 
goldfish, there are many work to do, such as change water and supplement oxygen by using pumps and air pumps two kinds of equipment. But, these devices are through human switch control, inconvenient operation. Many researchers have studied intelligent fish tanks, although some research results, but still some shortcomings. Hence, in this paper, for these shortcomings, we designed a microcontroller-based intelligent fish tank control system.

\section{The Design of Intelligent Fish Feeding System}

\subsection{Project design}

The GY201 photosensitive sensor module is applied to detect light intensity in this system. When the light is dark, the system can automatically control the lighting work. Two infrared sensors are used to detect the lowest water level and the highest water level of the fish tank. When the water level is lower than the lowest water level, the motor starts to add water. When the water level is higher than the highest water level, the water will be cut off automatically. The system clock is generated by timer timing, and feeding timing alarm clock is specified by keyboard setting. STC89C51 single chip micro-computer is used as the main control chip to complete sensor parameter acquisition, system automatic control and so on.

\subsection{Schematic diagram of the system}

The hardware composition of the system includes: minimum system of single chip microcomputer, feeding control circuit, water level control circuit, keyboard circuit, 1602 LCD circuit, power module, etc. The schematic diagram of the system is shown in Figure 2.1. 


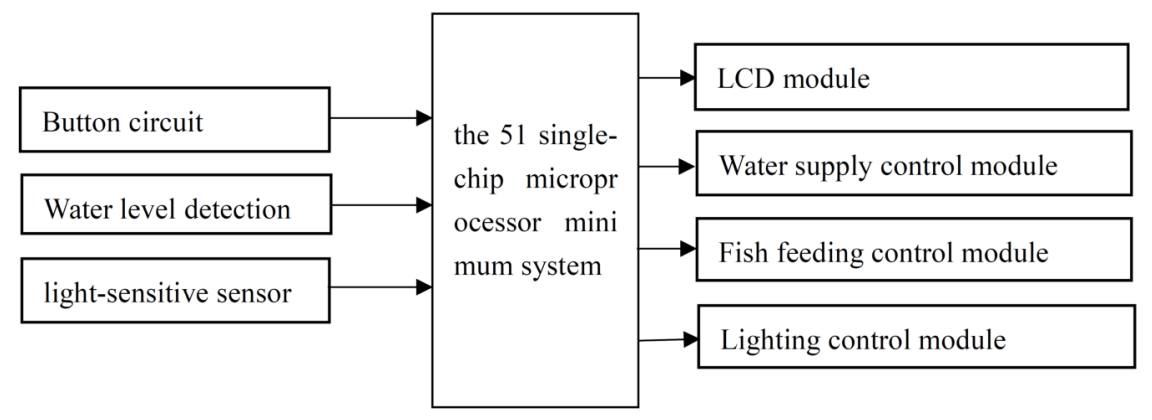

Figure 2.1. Schematic diagram of the system.

\subsection{Schematic diagram description of the scheme}

(1) Water level detection sensor module

The system uses HY01 infrared sensor to detect the lowest and highest water level of fish tank. One sensor is located at the low water level of the fish tank and one is located at the high water level of the fish tank.

(2) Lighting control module

The system uses GY201 sensitive sensor to detect the light intensity of the environment. When the light intensity is low, the system automatically outputs high level, and when the light is strong, the module outputs low voltage.

(3) Button circuit

This design uses 4 keys, e.g., K1, K2, K3, and K4 to complete the system parameters of the system setting. Among them, K1 is used to select the parameter to be set, K2 is used to set the parameter plus operation, K3 is used to set the parameter key operation, and K4 is used to set the parameter and exit the setting interface. 
(4) LCD module

1602 LCD is used to display the temperature data and other information. 1602 LCD is a relatively low-priced display device, which is enough for the information display of this subject.

(5) Water supply control

The system uses a relay switch to complete temperature control. The micro-controller only needs to output the IO level signal, you can control the relay break, and then control the operation of the temperature equipment.

(6) Fish feeding control

The system uses single chip microcomputer to drive UN2003 stepping motor driver chip to complete the driving task of stepping motor. Through the program control stepping motor forward and reverse to achieve the task of automatic feeding fish.

(7) Minimum system module of single chip microcomputer

The STC89C52 micro-controller is used as the control core, and the clock circuit and reset circuit constitute the minimum system module of single chip microcomputer. Complete the temperature and water level data acquisition, data processing, LCD display, control alarm and other functions.

\section{Functional Module Division}

\subsection{The design of single chip microprocessor minimum system}

The STC89C52 micro-controller is a 51 micro-controller with a standard 8051 core. The micro-controller chip is also the core of the traditional standard 51 micro-controller. There are still timing/counter, serial communication module, basic IO resource module, internal RAM and ROM resource module. In the field of industrial design, the microcontroller is used to accomplish tasks such as the development of intelligent control. 
In addition, the STC89C52 micro-controller can still work stably and normally under the condition of ultra-low frequency. If its mode is set in idle mode, when the CPU stops working, it can still keep the timer/counter interrupt system in working state. Once an external interrupt is triggered, the system automatically resumes the outage and the micro-controller starts to work. The highest operating frequency is $35 \mathrm{MHz}$, where $6 \mathrm{t} / 12 \mathrm{t}$ is optional. The minimum system of single chip microcomputer is generally composed of 51 single chip microcomputer, power module, reset circuit and clock circuit.

\section{(1) Reset circuit design}

The reset of single chip microcomputer can be divided into two kinds: power on automatic reset and button manual reset. The power on reset circuit consists of $22 \mu \mathrm{F}$ electrolytic capacitor and $10 \mathrm{~K}$ resistor. At power up, the capacitor charging current is the maximum, the capacitor is equivalent to a short circuit, and the RST terminal is high level, which is reset automatically. When the voltage at both ends of the capacitor reaches the power supply voltage, the capacitor charging current is zero, the capacitor is equivalent to an open circuit, and the RST terminal is low level, so the program runs normally.

Generally, the key reset circuit consists of micro-switch, $10 \mathrm{~K} \Omega$ resistor and $1 \mathrm{~K} \Omega$ resistor with $10 \mu \mathrm{F}$ electrolytic capacitor. When manual reset is needed, by pressing the micro-switch and connecting two resistors to realize voltage division, the output will get a high voltage, which meets the conditions of single chip microcomputer reset. When the hand is released, it is low voltage if the voltage is not obtained during reset and disconnection, so the single chip microcomputer will not be reset. Here we use power on reset to achieve. The circuit is shown in Figure 3.1. 


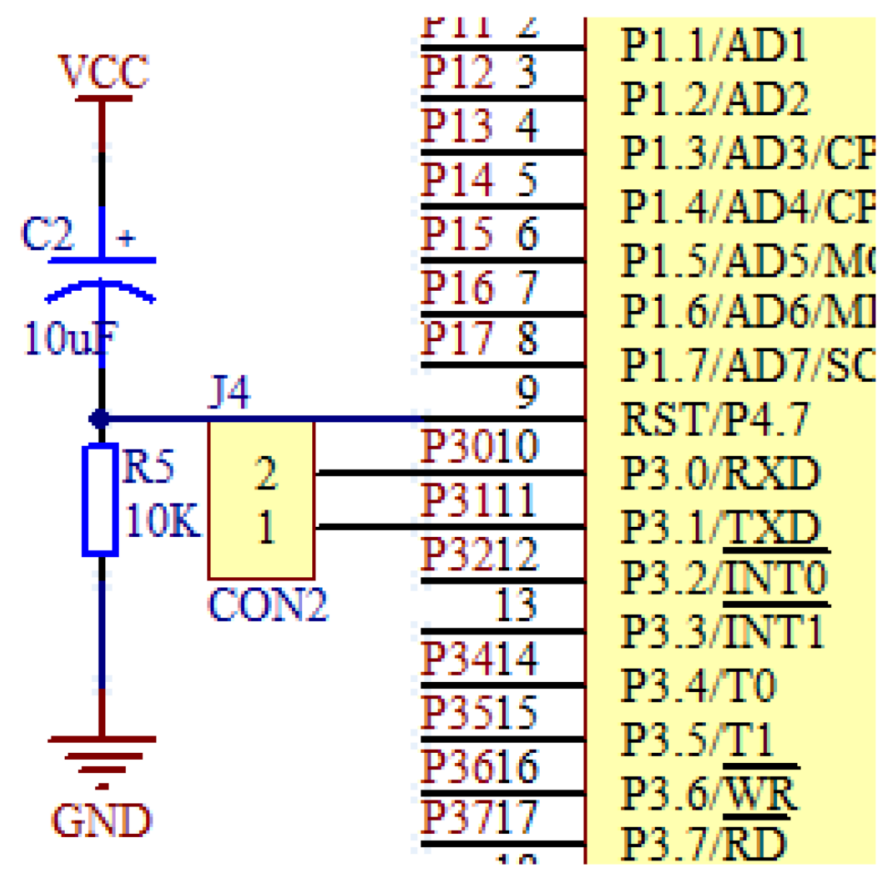

Figure 3.1. Reset circuit.

(2) Clock circuit design

To work, a single-chip computer must have a clock circuit. The clock circuit plays a role in providing the basic clock beat for the operation of the single-chip computer in the minimum system of the single-chip computer. If there is no clock circuit in single chip microcomputer, it can not work normally. According to different clock circuits, it can be divided into internal clock and external clock. In MCS-51 chip, there is a high gain inverting amplifier used to form oscillator. Its input is chip pin XTAL1, and its output is pin XTAL2. On the outside of the chip, XTAL1 and XTAL2 are connected with crystal oscillator and fine-tuning capacitor to form a stable self-excited oscillator, which outputs about $3 \mathrm{~V}$ sine wave on pins XTAL1 and XTAL2, which is the oscillation circuit of single chip microcomputer. As shown in Figure 3.2. 


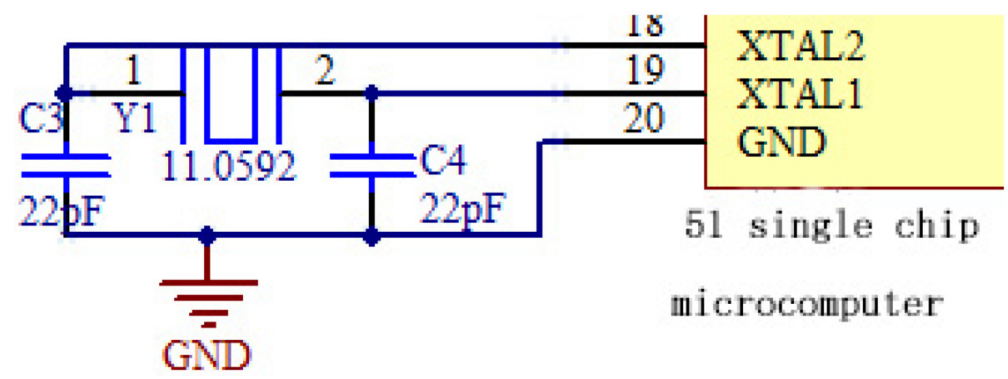

Figure 3.2. System clock circuit.

Generally speaking, the capacitance $\mathrm{C} 1$ and $\mathrm{C} 2$ are about $20 \mathrm{pF}-30 \mathrm{pF}$, which is mainly used to help the oscillator vibrate. The frequency range of crystal oscillation is $1.2 \sim 12 \mathrm{MHz}$. The frequency range of the external crystal oscillator of the single-chip microcomputer can be between $1 \mathrm{MHz}$ and $12 \mathrm{MHz}$. The higher the frequency, the faster the single-chip microcomputer will run. But it can't be higher than $12 \mathrm{MHz}$. Because the frequency is higher than the upper limit, the clock signal can't be collected inside the single-chip microcomputer. It is beyond the maximum range of single chip microcomputer frequency acquisition.

\subsection{The design of button circuit}

The system adopts four keys as the input mode of parameter setting. Where $\mathrm{K} 1$ is used to set and select the parameters, and $\mathrm{K} 4$ is used to open and close the alarm. The interface circuit with the micro-controller is shown in Figure 3.3. 


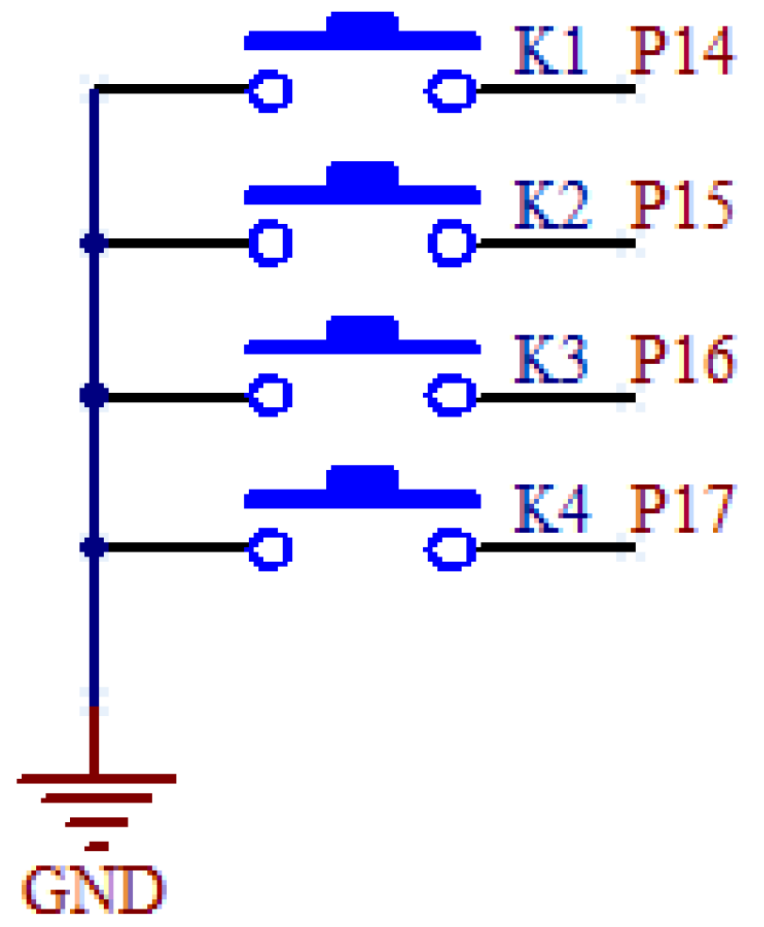

Figure 3.3. Keyboard circuit diagram.

\subsection{The design of LCD1602 LCD circuit}

Character LCD is a kind of dot matrix LCD specially used to display letters, numbers, symbols and so on. LCD1602 LCD is adopted in this design. Its overall dimension is $80 \times 36 \times 14$, single $5 \mathrm{~V}$ power supply, 16 pins from left to right. The CGROM inside the 1602 LCD module has stored 160 different dot matrix character graphics, including Arabic numbers, English letters, common symbols, Japanese kana, etc. Each character has a fixed code, such as the code of the capital English letter " $\mathrm{A}$ " is $01000001 \mathrm{~B}(41 \mathrm{H})$, and the module will display the address $41 \mathrm{H}$. LCD1602 liquid crystal display module can be directly connected with STC89C52. DB0-DB7 can be connected with P00-P07 for data transmission and reception, 4, 5 and 6 pins can be connected with P2.7, P2.6, and P2.5 of STC89C51. The circuit is shown in Figure 3.4. 


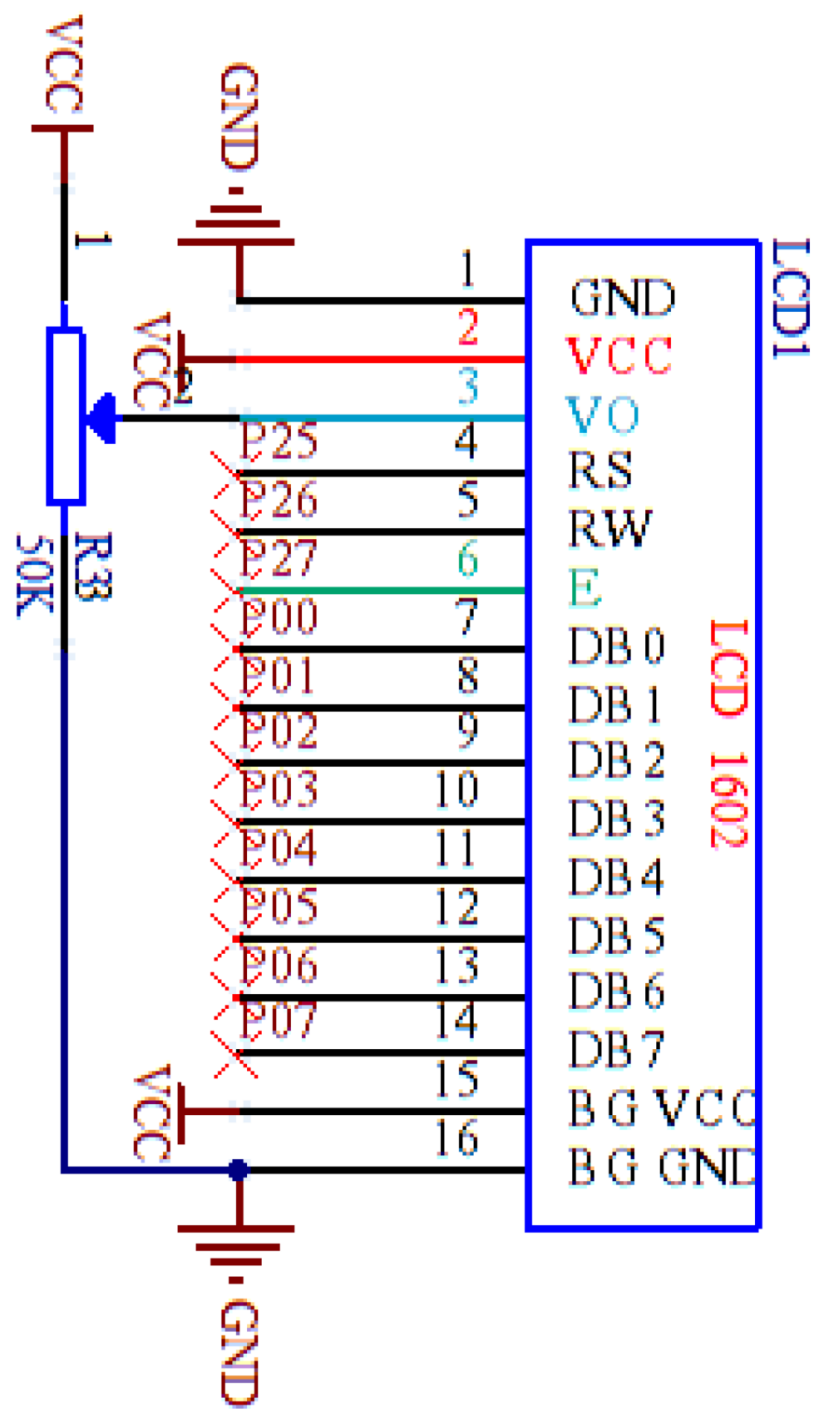

Figure 3.4. LCD drive circuit diagram.

\subsection{Circuit design of buzzer}

Working principle of alarm circuit: designed with alarm function, the alarm is sent out by buzzer. The triode plays the role of switch. When the voltage of the base is high, the triode is on and the buzzer is activated. When the voltage of the base is low, the triode is cut off and the buzzer 
cannot be activated. The voltage of the base pole is controlled by the output of the I/O port of the single chip microcomputer. The resistance is used for current limiting protection components. The circuit diagram of buzzer is shown in Figure 3.5.

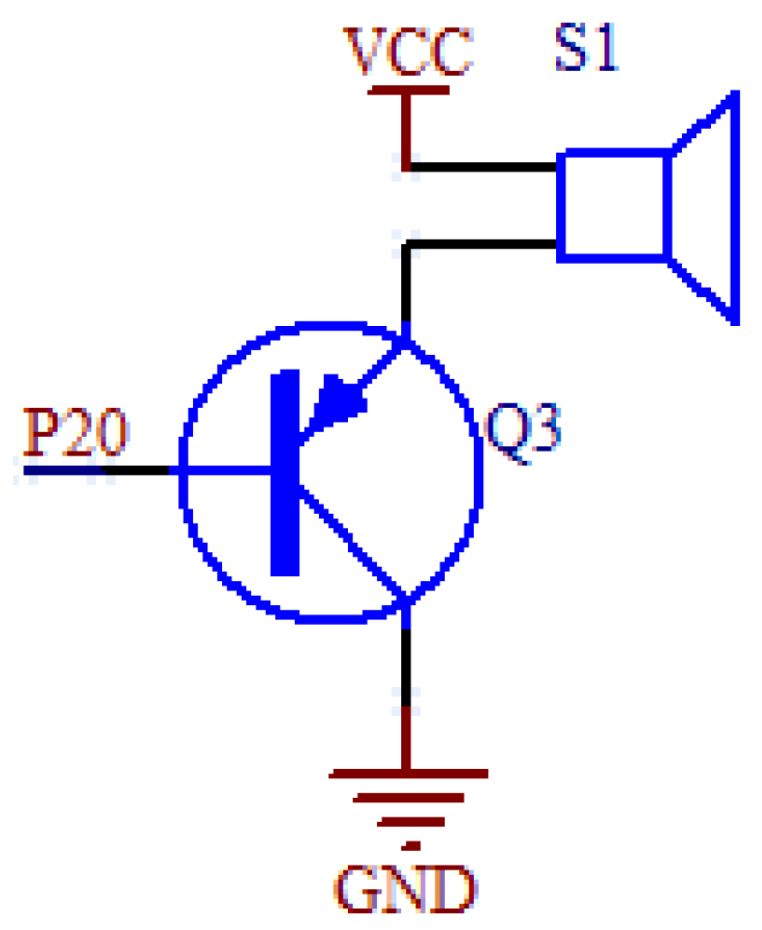

Figure 3.5. Circuit diagram of buzzer.

\subsection{Automatic feeding control circuit}

ULN2003 has high working voltage, high working current, filling current up to $500 \mathrm{~mA}$, and can bear $50 \mathrm{~V}$ voltage when it is closed, and the output can also run in parallel with high load current. For each pulse input, the output shaft of the rotary stepping motor rotates once. Stepping motor is a kind of induction motor. Its working principle is to use electronic circuit to supply power when DC is changed into components. The current is controlled by multi-phase sequence. When this current is used to supply power for stepping motor, the stepping 
motor can work normally. The driver is a multi-phase sequence controller that supplies power for stepping motor in time. In this system, UN2003 chip is used to drive stepping motor. It uses four I/O ports of single chip microcomputer, $\mathrm{P} 10 \backslash \mathrm{P} 11 \backslash \mathrm{P} 12 \backslash \mathrm{P} 13$ to complete the drive task. The driving circuit diagram of stepping motor is shown in Figure 3.6.

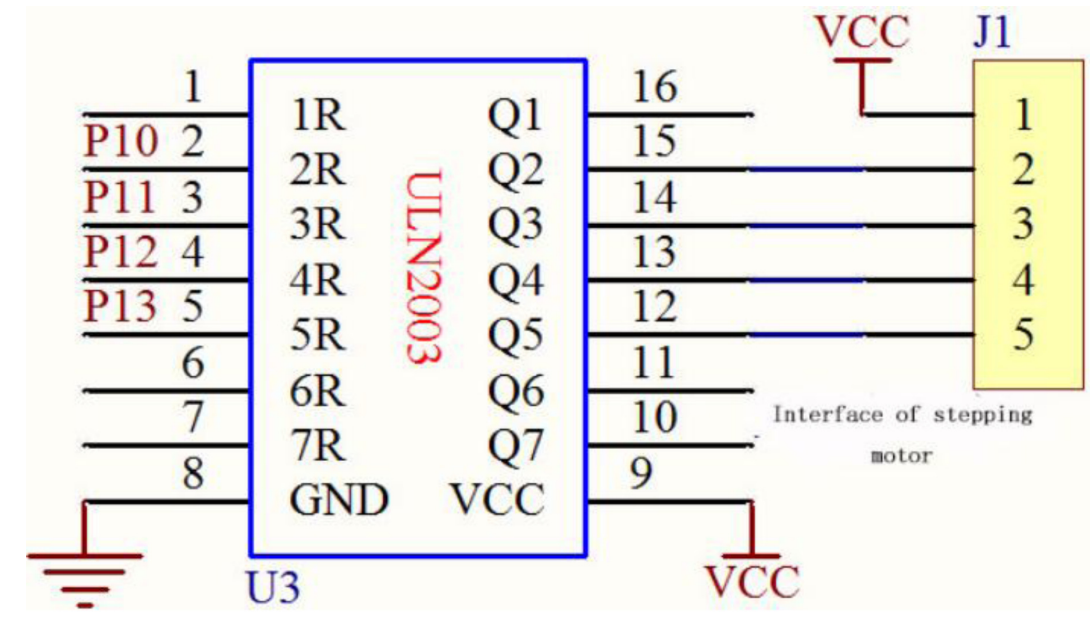

Figure 3.6. Schematic diagram of motor drive module.

\subsection{Water supply control circuit}

(1) Relay action

Relay is an automatic switching element with the isolation function. It is widely used in remote control, telemetry, communication, automatic control, mechanics and power electronic equipment, and is one of the most important control elements. Relays generally have sensing mechanisms (input slots) that reflect certain input variables. An actuator (output part) capable of "on" and "off" control of the controlled circuit. There is also an intermediate mechanism (drive part) that couples the input, handles functions, and drives the output part. 
(2) Application of relays in this system

The system uses relay control, water supply equipment on and off to realize the dynamic control of water level in the fish tank. Because the I/O port of single-chip microcomputer can not directly drive the relay, the triode S8550 is used to drive the relay. The circuit diagram is shown in Figure 3.7.

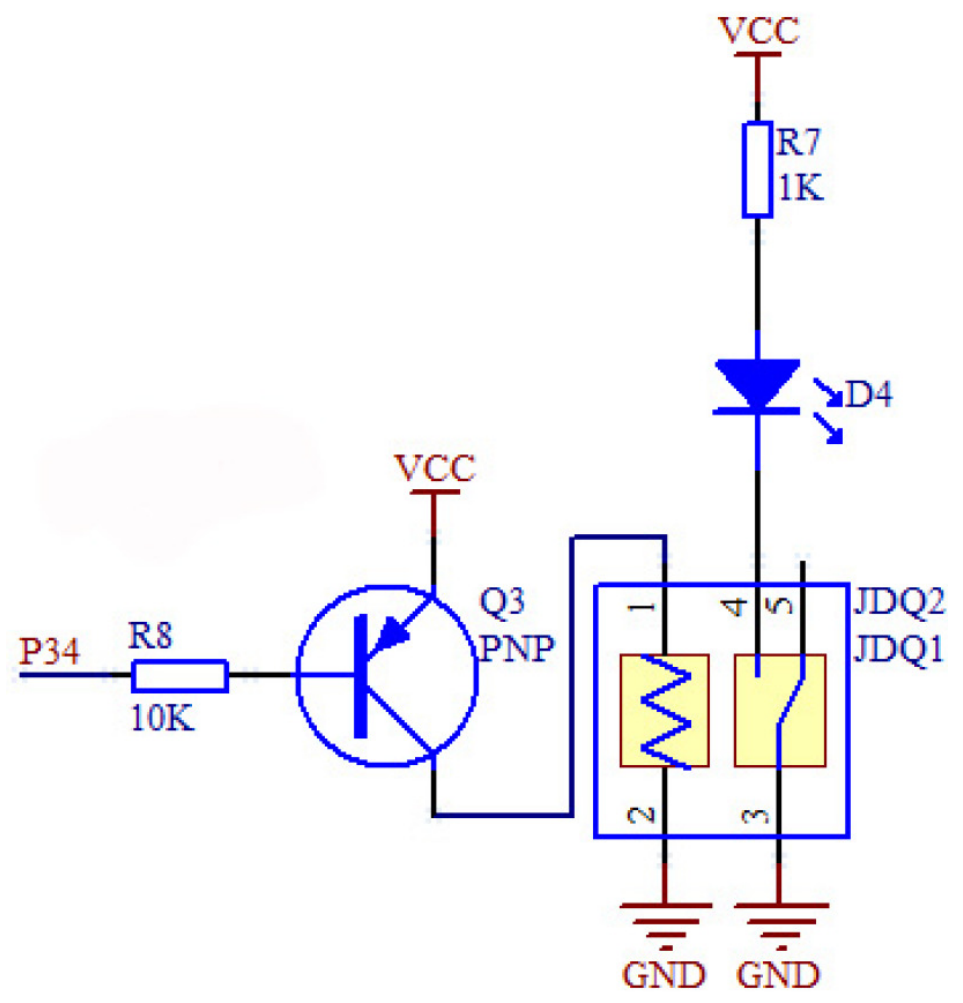

Figure 3.7. Design of driving circuit of water supply control switch.

\section{System Programming}

\subsection{System main program design}

After the system is powered on, initialization is carried out first to complete initialization tasks such as LCD, keyboard, etc. Then into the dead cycle, the single-chip microcomputer controls the DS18B20 sensor to 
collect the temperature data in the fish tank environment, and converts, according to the data calculation temperature control. The function of automatic antivirus is achieved by timing. Control the light of fish tank through light detection. The procedure flow chart is shown in Figure 4.1.

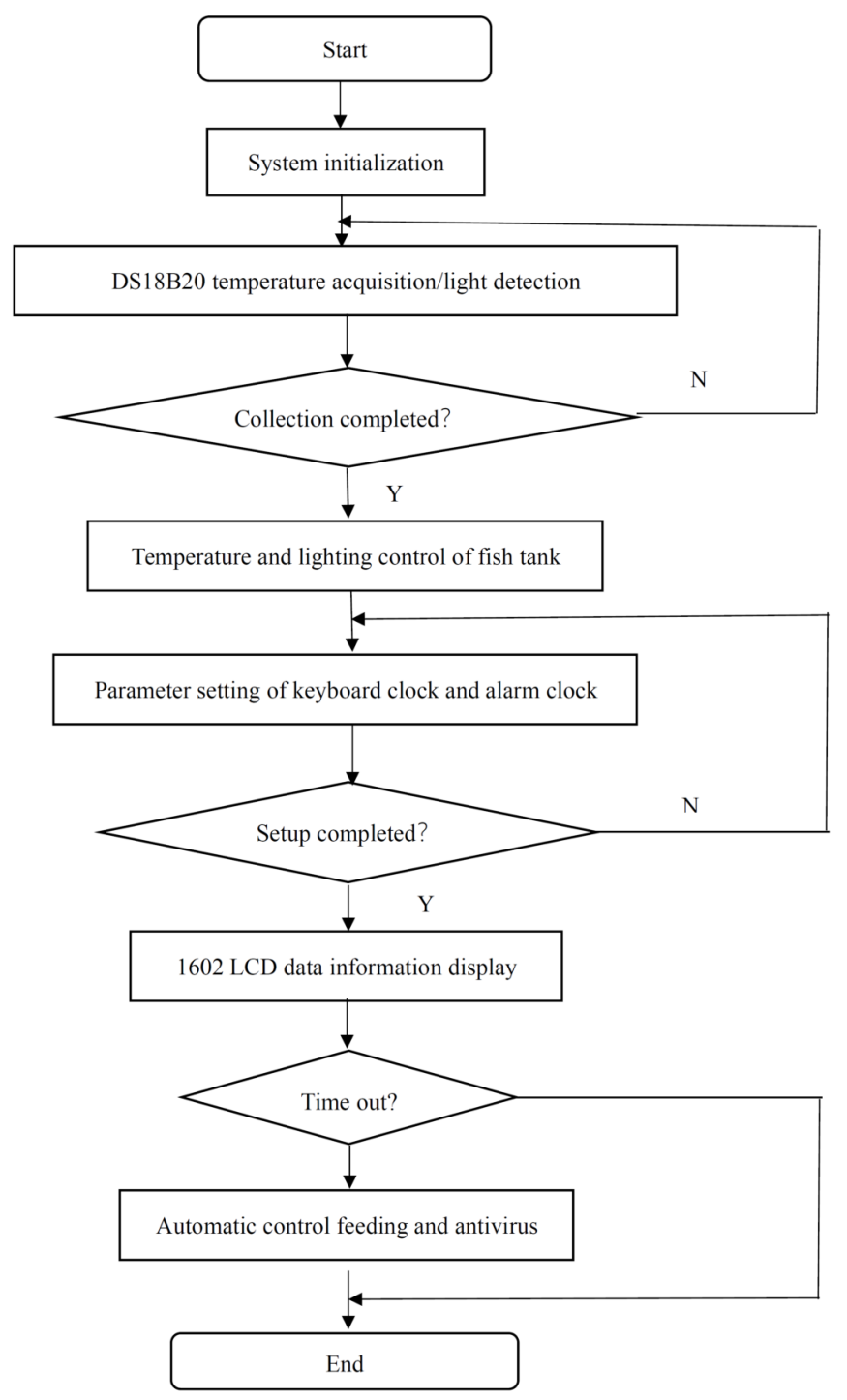

Figure 4.1. The main program flow chart of the system. 


\subsection{LCD1602 LCD display subroutine}

LCD is driven in much the same way. Generally, it needs to be initialized before use to ensure that it is ready for display; then the general operation is to write commands and read data, which are common operations. The procedure flow chart is shown in Figures 4.2 and 4.3.

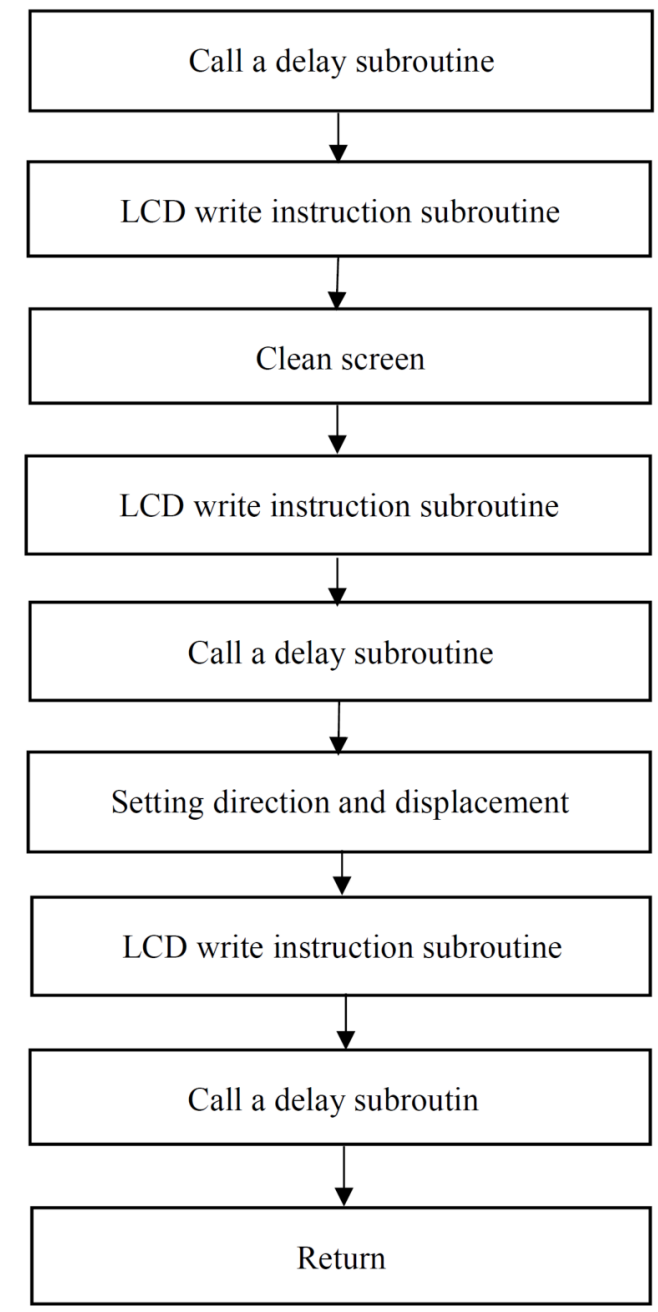

Figure 4.2. Flow chart of writing instruction subprogram. 


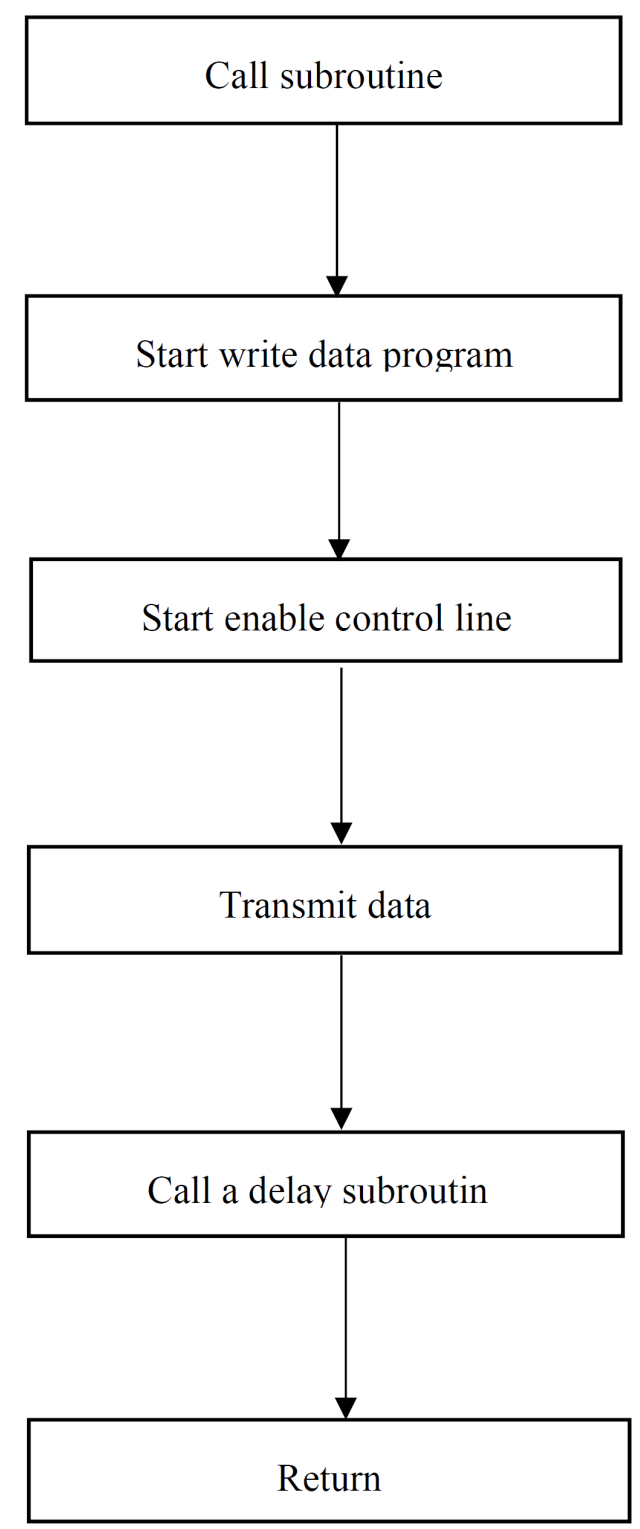

Figure 4.3. Flow chart of writing data subprogram.

The process of LCD displaying character information generally requires the following steps:

Step 1. Initialize the LCD to prepare for display; 
Step 2. Set the display coordinates, for example, you need to set the position when you need to display the lines and columns;

Step 3. Output the display Output display is to think about whether to display a single character, string or number. Call the corresponding function. Of course, the most used in the LCD process is to write instructions and data, LCD busy detection and so on.

\subsection{Alarm subprogram design}

Alarm alerts for this system are implemented through buzzers. According to the principle of alarm circuit, the alarm is realized by an I/O port. When the I/O port outputs a low level, the buzzer will sound. When the I/O port outputs a high level, the buzzer does not sound. The flow of alarm prompt subprogram is as follows.

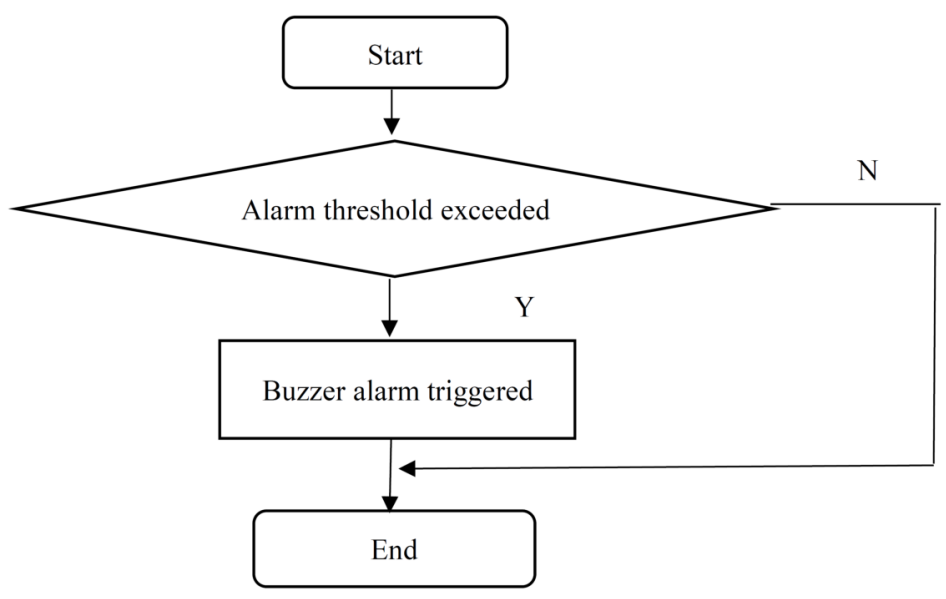

Figure 4.4. Flow chart of buzzer/LED alarm subprogram.

\subsection{Key recognition subprogram design}

In this design, the keyboard is used to realize the input operation of human-computer interaction. Analyzing from the structure, the keyboard is directly connected to the four I/O ports P3.4, P3.5, P3.6, and P3.7 of the single chip microcomputer. Then the other end of the key is directly connected to GND. The idea of keyboard program recognition: First, scan to see which I/O has a key press, if there is a key press, then determine 
which row is and record a value. Second scan to determine which column is the key press. Third you will know exactly which key is pressed, and finally the only key code will be obtained. The procedure flow chart is shown in Figure 4.5.

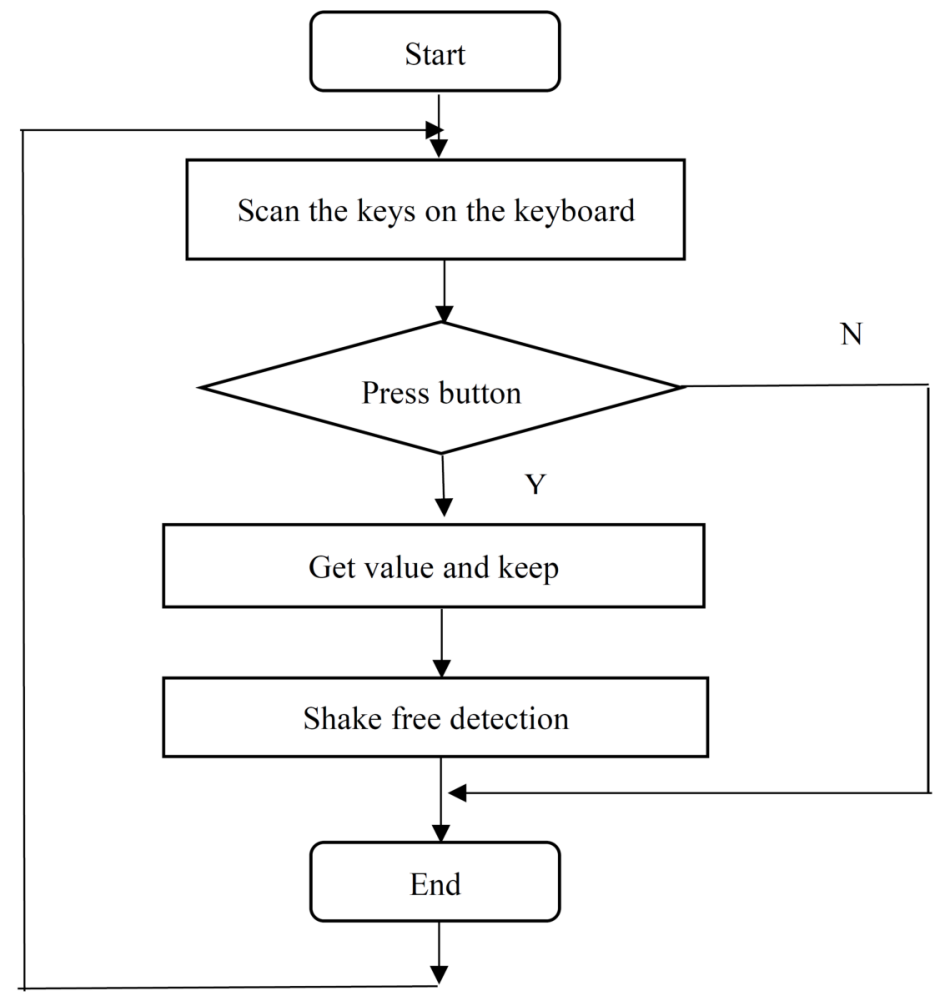

Figure 4.5. Flow chart of key control subprogram.

\subsection{Feeding subprogram design}

Read the system clock in real time after the system is powered on. At the same time, scan whether the alarm time is up. If so, start the stepper motor to complete the feeding control. This system uses relay to control the opening and closing of feeding switch. Because the I/O port of singlechip microcomputer cannot directly drive the relay, it uses the triode S8550 to drive the relay. The procedure flow chart is shown in Figure 4.6. 


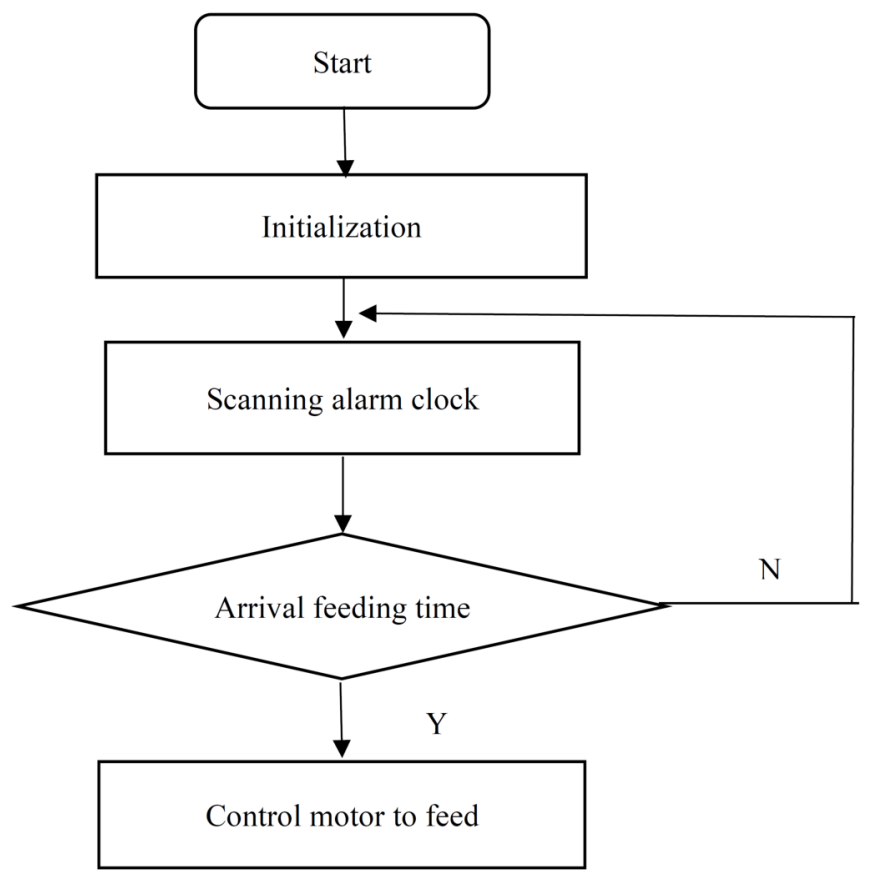

Figure 4.6. Flow chart of antivirus and feeding control.

\section{Operation Steps}

The system uses HY01 infrared sensor to detect the lowest and highest water level of fish tank. One sensor is located at the low water level of the fish tank and one is located at the high water level of the fish tank.

The system uses GY201 sensitive sensor to detect the light intensity of the environment. When the light intensity is low, the system automatically outputs high level; when the light is strong, the module outputs low voltage.

This design uses 4 keys K1, K2, K3, and K4 to complete the system parameters of the system setting. Among them, K1 is used to select the parameter to be set, $\mathrm{K} 2$ is used to set the parameter plus operation, K3 is used to set the parameter key operation, and K4 is used to set the parameter and exit the setting interface. The results are shown in Figures 5.1-5.4. 


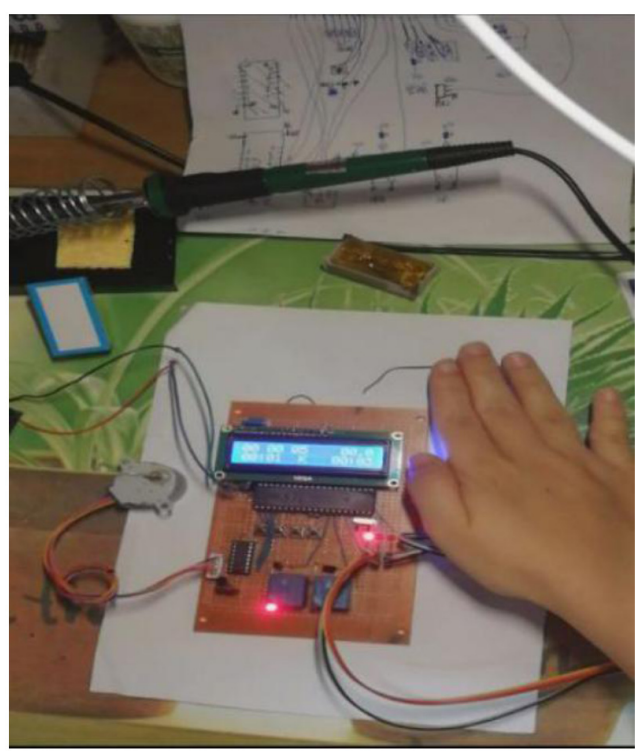

Figure 5.1. When no light is sensed, the system will automatically illuminate.

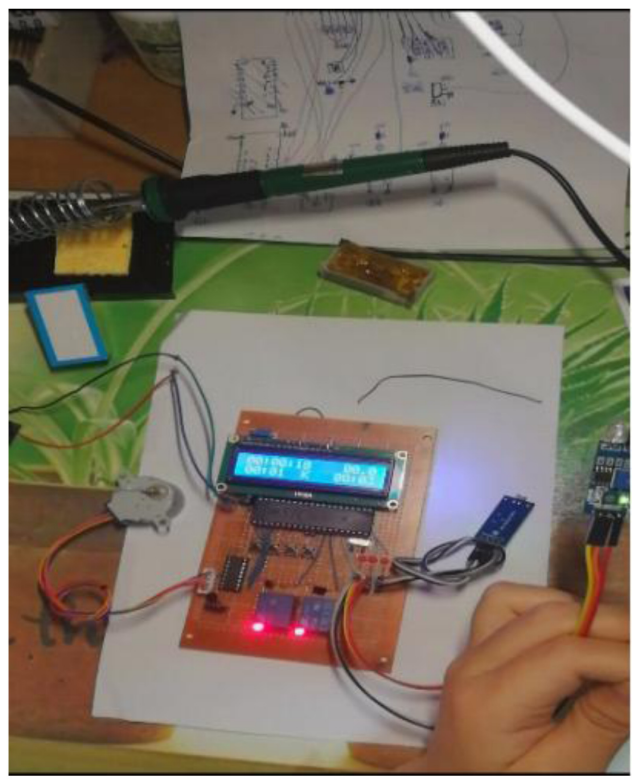

Figure 5.2. When there is an induction at the lower water level, water will be added automatically. 


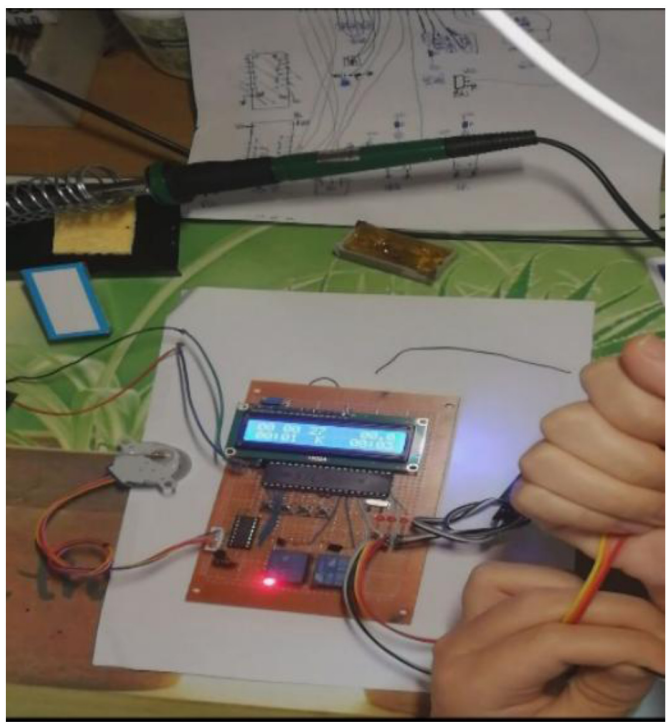

Figure 5.3. Stop adding water when the upper water level is sensed.

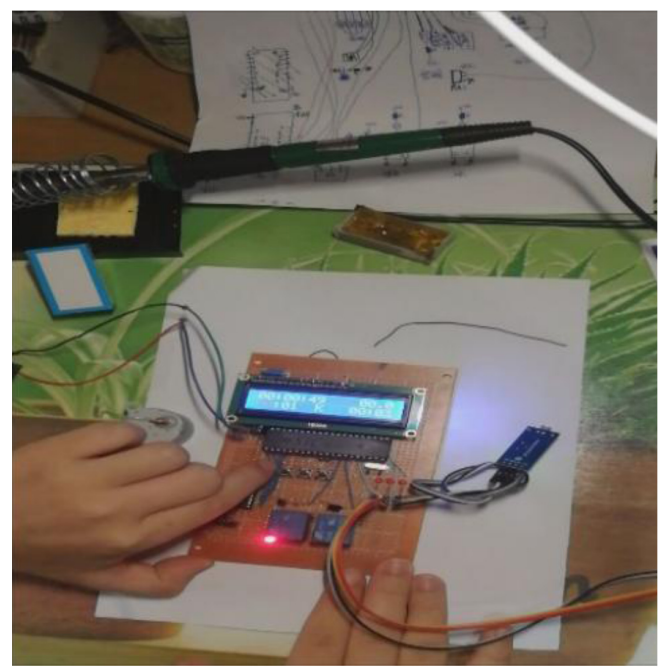

Figure 5.4. Adjust the time through the button. 


\section{Conclusion}

With the improvement of people's living standards, people's spiritual pursuit is getting higher and higher. The intelligent life has become an unstoppable trend. The automatic feeding system of fish is produced in this context. The intelligent fish tank system based on the microcontroller designed by this topic has the following advantages:

(1) The design solves the intelligent problem of traditional fish tank

Traditional intelligent fish tank products are simply done by hand to control the fish tank, with little involvement in electronic technology. Large fish tank generally uses remote control mode control switch, but there is a great problem in humanization, cannot achieve automatic control. The intelligent fish tank products designed by this topic solve the above problems and realize the functions of automatic feeding, automatic water addition, and automatic lighting.

(2) Make smart fish tank products smarter and less expensive

The intelligent fish tank designed by this system applies electronic technology and intelligent control technology to the fish tank, making it have intelligent functions such as lighting, water change, and feeding, which is more intelligent and user-friendly than the traditional fish tank. The technology used in this product can also be applied to other areas of electronic products, so that products become more intelligent, effectively reduce the cost and volume of existing positioning products, more conducive to product integration.

The system can not only reduce the manpower needed to maintain the landscape, but also realize the intelligent cultivation and reduce the cost of human and material resources in life. The intelligent feeding system of fish has great application value in life, business, and industry. The technology applied in this product can also be more applied to other fields of electronic products, so that the products become more intelligent.

\section{Acknowledgement}

This research is supported by Zhilian Science and Innovation Workshop, the Research topic of teaching reform (No.18055), the project of scientific research of Jining Medical University (Nos. 600529001 and 2018JYQD03). 


\section{References}

[1] G. J. Snyder and E. S. Toberer, Complex thermoelectric materials, Nature Materials 7(2) (2008), 105-114.

DOI: https://doi.org/10.1038/nmat2090

[2] H. Sasabe and J. Kido, Development of high performance OLEDs for general lighting, Journal of Materials Chemistry C: Materials for Optical and Electronic Devices 1(9) (2013), 1699-1707.

DOI: https://doi.org/10.1039/c2tc00584k

[3] M. Antonelli, G. Conti, P. Pelosi, C. Gregoretti, M. A. Pennisi, R. Costa, P. Severgnini, M. Chiaranda and R. Proietti, New treatment of acute hypoxemic respiratory failure: Noninvasive pressure support ventilation delivered by helmet: A pilot controlled trial, Critical Care Medicine 30(3) (2002), 602-608.

[4] Y. Xu, H. Bai, G. Lu, C. Li and G. Shi, Flexible graphene films via the filtration of water-soluble noncovalent functionalized graphene sheets, Journal of the American Chemical Society 130(18) (2008), 5856-5857.

DOI: https://doi.org/10.1021/ja800745y

[5] C. Schmidt and A. Goetzberger, Single-tube integrated collector storage systems with transparent insulation and involute reflector, Solar Energy 45(2) (1990), 93-100.

DOI: https://doi.org/10.1016/0038-092X(90)90033-9

[6] J. J. H. Liou, G.-H. Tzeng and H.-C. Chang, Airline safety measurement using a hybrid model, Journal of Air Transport Management 13(4) (2007), 243-249.

DOI: https://doi.org/10.1016/j.jairtraman.2007.04.008

[7] K. J. Shin and A. V. Angani, Development of water control system with electrical valve for smart aquarium, International Conference on Applied System Innovation (2017), 428-431.

DOI: https://doi.org/10.1109/ICASI.2017.7988444

[8] T. Citarasu, Herbal biomedicines: A new opportunity for aquaculture industry, Aquaculture International 18(3) (2010), 403-414.

DOI: https://doi.org/10.1007/s10499-009-9253-7

[9] C. Dongyang, Z. Zihan, C. Xinrong and T. Jintian, Intelligent bus measurement and control system design based on the TEDS, IERI Procedia 3 (2012), 22-27.

DOI: https://doi.org/10.1016/j.ieri.2012.09.005 